\title{
L'Éducation des filles congolaises au maquis de Mulele : Arme de libération ou force $\mathrm{d}^{\prime}$ (auto)destruction?
}

\author{
Annette Lembagusala Kikumbi \\ KU Leuven
}

Marc Depaepe

KU Leuven

RÉSUME

Depuis ses origines remontant au temps colonial, l'école congolaise est marquée par l'absence d'une relation dialectique "École-Société " pouvant aboutir à un système harmonieux. Cette carence entraine la déculturation et le dualisme culturel au sein des structures éducatives. L'école maquisarde instituée par Mulele dans le Kwilu, présente un projet d'éducation compatible à la culture africaine. Dans cet article, nous donnons la voix aux maquisards (élèves et enseignants) qui racontent leur vécu durant les années de la rébellion; expériences qui sont qualifiées par les auteurs selon leur contexte historique et leurs mérites éducatifs. Notre question centrale consiste à savoir les effets de cette insurrection sur l'éducation des filles. Qu'est-ce que la révolution leur a apporté de nouveau? Comment s'organisaient les enseignements dans le maquis? À quoi le rejet de la culture occidentale a-t-il abouti? Pour répondre à ces questions, nous nous servons des expériences scolaires de 20 élèves et 12 enseignants qui soulignent les enjeux majeurs de l'éducation maquisarde. Nous nous limitons à la province du Kwilu, siège de la rébellion muleliste. En plus des entretiens, nos sources proviennent des archives des Sœurs Annonciades d'Heverlee et du carnet de notes tenu par un chef d'équipe des maquisards rencontré à Kikwit.

\section{ABSTRACT}

Since its inception in the colonial period, the Congolese school has been characterised by the absence of a "School-Society" dialectical relationship, which could potentially lead to a harmonious system. This deficiency leads to deculturation and cultural dualism within educational structures. The "resistance" school set up by Mulele in the Kwilu region offers an educational approach that is compatible with African culture. In this article, we let members of the maquis (students and teachers) talk about their experiences during the years of the rebellion; experiences that are qualified by the authors against their historical background and educational merits. Our key question concerns the effects of the insurrection on the education of girls. What new elements did the revolution bring them? How was teaching organised in the maquis? 
What did the rejection of Western culture lead to? To answer these questions, we look at the experiences of 20 pupils and 12 teachers which highlight the major issues facing education in the maquis. Here, we limit ourselves to the province of Kwilu, the heart of the rebellion led by Mulele. In addition to the interviews, our sources include the archives of the Annonciades Sisters of Heverlee and the notebook kept by a team leader of the maquisards met in Kikwit.

\section{Introduction : Situation de l'étude}

"Le Lumumbisme est une doctrine, tandis que le Mulelisme est une force. Les deux éléments conjugués donnent une arme invincible pour délivrer le peuple congolais de la servitude des impérialistes ». C'est sur ces mots que s'ouvrait en 2003 le premier numéro du journal Révolution Congolaise, consacré à Pierre Mulele (1929-1968), " éducateur, organisateur et libérateur des masses populaires du Congo ». ${ }^{1}$ Le présent article s'efforce de déterminer dans quelle mesure ce mouvement est parvenu à atteindre ses objectifs.

Cette question, qui sera clarifiée au fil du présent article, entre dans le cadre d'un doctorat sur l'éducation des filles dans la province du Kwilu ${ }^{2}$ et est en soi le corollaire d'un programme de recherche sur l'histoire de l'enseignement colonial et postcolonial entamé dans les années 1990 à la KU Leuven. ${ }^{3}$ L'historiographie du passé colonial belge a jadis été bafouée, ${ }^{4}$ une sentence qui s'appliquait encore plus à l'historiographie pédagogique qu'à l'histoire générale, et certainement à celle de l'éducation des filles congolaises, qui n'avait encore jamais fait l'objet d'une étude systématique. ${ }^{5} \mathrm{Il}$ est évident que des études de cas régionales constituent un bon point de départ pour combler cette lacune et d'autres. Comme l'a très justement dit l'influent historien congolais Isidore Ndaywel è Nziem :

Les connaissances actuellement disponibles ont été accumulées essentiellement à partir d'une démarche "par le haut ", rapportant surtout l'histoire des " grands hommes " et des "faits saillants" se déroulant dans la capitale. L'histoire "d'en bas ", celle du peuple reste à faire, de même que l'histoire des différentes régions. Jusqu'à présent, les informations disponibles sur le Congo sont à la fois discriminatoires et inégalement réparties sur le plan spatial. ${ }^{6}$

Parallèlement au besoin intrinsèque d'apprendre à mieux connaître l'histoire de l'éducation et de l'enseignement au Congo — une approche qui, si elle voulait aboutir à une interprétation équilibrée et nuancée, n’impliquait pas seulement l'engagement de chercheurs belges, mais aussi congolais ${ }^{7}$ — ce projet de recherche plus vaste a également poursuivi dès le départ l'appréhension de conceptions théoriques sur la transmission culturelle générale entre les générations. Une question essentielle à ce niveau portait sur les effets résiduels des objectifs de formation largement imposés, comme dans le cadre de l'offensive missionnaire menée au Congo belge. ${ }^{8}$ Tous ceux qui ont l'habitude des archives scolaires savent qu'il n'est pas toujours aisé de répondre à cette question. En dehors des informations normatives contenues dans les traités, règlements d'école, plans d'enseignement, etc., sur la question du " comment " il 
convenait d'éduquer (c'est-à-dire en tendant à quel idéal), on reste généralement sur sa faim lorsqu'il s'agit de savoir ce qui s'est effectivement fait sur le terrain pédagogique. ${ }^{9}$ Dans le cadre de la recherche sur les effets des expériences scolaires dans la colonie, nous avons ainsi été assez rapidement contraints de nous tourner vers l'histoire orale ${ }^{10}$ — une technique que le contexte africain ne permet pas toujours d'appliquer de manière orthodoxe. La prédominance de la culture orale, avec ses propres schémas de perception circulaire du temps et ses descriptions parfois amplifiées ou ses exagérations dans le souvenir, joue ici un rôle important, ${ }^{11}$ tout comme le fait non négligeable que le Congo est aujourd'hui encore en proie à différents flux migratoires et presque constamment pris dans des conflits internes et étrangers. $\mathrm{Vu}$ ces circonstances, il n'est pas toujours évident de suivre à la lettre les prescriptions méthodologiques eurocentriques de l'historiographie orale. Il faut, au demeurant, pouvoir bien sûr se servir des langues locales, ce qui implique une connaissance suffisante du terrain. ${ }^{12}$

Il en va de même dans la présente recherche pour laquelle, parallèlement à la consultation d'égo-documents (essentiellement des témoignages contenus dans des lettres de missionnaires, ${ }^{13}$ mais aussi les notes d'un muleliste), ${ }^{14} 32$ personnes ont été interviewées : 20 élèves et 12 enseignants ayant vécu au maquis pendant la période de la rébellion. Beaucoup de Congolais qui ont vécu la rébellion muleliste en gardent des souvenirs indélébiles. Il en ressort une peur inouïe et énormément de frustration. C'est pour cette raison qu'il nous a été très difficile de trouver des informateurs disponibles pour nous relater leurs souvenirs. ${ }^{15}$ En outre, ceux qui ont vécu dans le maquis acceptent à peine de dévoiler les secrets de la rébellion. D’autres maquisards ne se dévoilent pas par peur de représailles.

Pour recueillir nos données, nous visitions un premier maquisard, qui après l'entrevue, nous en indiquait un autre. Nous avons procédé de la même manière jusqu'à atteindre un nombre jugé suffisant (que nous avons évalué à environ 30 personnes). Au départ, nous avons voulu utiliser le simple échantillon aléatoire qui consiste à extraire au hasard un nombre fixe de sujets. Vu les difficultés de trouver des individus disponibles, nous avons recouru à l'échantillon occasionnel. La sélection des répondants a été basée sur deux critères : l'expérience et la disponibilité des informateurs.

Les entretiens ont été menés à partir d'un guide d'entrevue de cinq items élaborés à l'avance. ${ }^{16}$ Pour chaque entretien, nous avons accordé une heure. Nous nous sommes servis d'un dictaphone comme moyen d'enregistrement. Après audition, nous les avons transcrits sur différents fichiers. Pour résoudre le problème de la " représentativité » comme écueil principal de la méthode de l'histoire orale, nous avons tenu compte de la qualité de l'information que détenaient les différents maquisards lors de nos entretiens.

Bien que des préférences personnelles et des éléments subjectifs puissent bien sûr encore intervenir dans notre interprétation - l'auteure principale étant elle-même religieuse chez les Annonciades - nous pensons toutefois que le présent article demeure pertinent en allant au-delà des perceptions particulières. Au fil des ans, notre étude de la situation congolaise nous a de plus en plus convaincus que la structure et les effets de la vie pédagogique " moderne ", y compris les défaillances systémiques, 
sont les plus visibles dans un contexte (post)colonial où, partant souvent d'une croyance assez naïve dans la suprématie de la culture occidentale (lire catholique), il fallait en quelque sorte transmettre/reproduire cette modernisation dans son intégralité. Pour notre usage interne, nous avons qualifié ce processus de " cuisson sous haute pression " — une pensée en outre susceptible d'étendre l'intérêt pour l'histoire de l'éducation coloniale belge bien au-delà de la perspective nationale. ${ }^{17}$ Mais avant de pouvoir passer à ce genre d'hypothèses, il fallait bien entendu disposer d'évidences suffisantes. Comme c'est généralement le cas avec la recherche historique, l'ouvrage précède obligatoirement la théorisation ${ }^{18}$ — un constat qui vaut certainement aussi pour la question de savoir si le régime révolutionnaire de Mulele a eu ou non des effets " émancipatoires ». À notre connaissance, cette question n'a pas encore été explicitement posée, en dépit des tentatives d'auteurs tels que Benoit Verhaegen, ${ }^{19}$ Ludo Martens, ${ }^{20}$ Renée Fox, Willy De Craemer et Jean-Marie Ribaucourt ${ }^{21}$ de valoriser les qualités potentielles de l'idéologie sous-jacente.

Avant de nous risquer à émettre un avis nuancé sur cette question, nous allons successivement aborder les contextes politiques dans lesquels les idées de Mulele ont mûri; sa vision pédagogique en général et ses conséquences sur l'éducation des filles; et, bien sûr, ce qu'il en est ressorti en réalité, en accordant une attention particulière à la relation avec le système éducatif africain traditionnel d'une part et à la confrontation avec les reliquats du système d'enseignement colonial belge d'autre part.

\section{Contexte politique après l'indépendance}

Lors de son accession à la souveraineté nationale, le 30 juin 1960, le Congo était un immense pays s'étendant sur une superficie de $2345000 \mathrm{~km}^{2}$, soit environ 77 fois la taille de la Belgique qui avait jusqu'alors souvent considéré sa " colonie " comme une " dixième " province. La position stratégique du Congo au cour de l'Afrique et l'attrait de ses richesses naturelles le prédisposaient à un rôle de meneur. Malgré le peu de diplômés universitaires que recelait le pays, il paraissait possible de se développer. Cependant, peu de temps après l'indépendance, le pays sombra dans une instabilité politique indescriptible. Cette instabilité était évidente dans la sphère gouvernementale, se traduisant par des querelles et des tiraillements continuels entre les dirigeants des différents partis politiques en place. Dans ce climat chaotique, le pays ne pouvait que sombrer dans des conflits provoquant profonde anarchie dans l'ensemble du pays : guerres civiles, mutineries des forces armées, sécession de certaines provinces, dislocation brutale des structures administratives, insécurité généralisée. ${ }^{22}$

Le 11 juillet 1960, Moïse Tshombe, le chef du parti de la Confédération des associations tribales du Katanga (CONAKAT), se proclama président du Katanga "indépendant ». Il fut soutenu par la Belgique avec qui il cherchait à s'associer pour garder le contrôle de l'Union minière du Haut-Katanga. Entretemps, les troubles ethniques éclatèrent entre les Katangais et les Baluba du Kasaï qui travaillaient dans les mines de l'administration coloniale. Deux mois plus tard, le 9 août 1960, Albert Kalonji, le cofondateur du Mouvement national congolais, suivit à son tour les traces de Tshombe et proclama l'indépendance du Kasaï. Il devint le président de « l'État autonome du 
sud-Kasaï » dont faisait partie le peuple Baluba. Le premier ministre Patrice Lumumba s'opposa de pied ferme à ces velléités séparatistes " en mobilisant tous les éléments nationalistes de l'armée congolaise contre les sécessionnistes de Katanga et du Kasaï. Mais le colonel Mobutu donna l'ordre à ses troupes d'arrêter leur offensive ». ${ }^{23}$ Dans cet imbroglio politique, le président Kasavubu décréta la dissolution du gouvernement de Lumumba le 5 septembre 1960. Du 12 au 14 septembre, le colonel Mobutu organisa, de son côté, son premier coup d'État contre le président de la République et le premier ministre. Dès lors, l'O.N.U. ne reconnaissait plus le gouvernement du premier ministre Lumumba. Le vice-premier ministre Antoine Gizenga entra alors en sécession, forma un gouvernement et installa le siège de son équipe gouvernementale à Kisangani. De son côté, Pierre Mulele, ${ }^{24}$ l'ex-ministre de l'Éducation nationale et des Beaux-Arts, s'exila en Égypte puis en Chine. Depuis l'étranger, " il amena plusieurs pays à reconnaître le gouvernement Lumumba-Gizenga ". ${ }^{25}$ Pendant son exil en Chine, Mulele suivit une formation militaire et politique avec l'intention de déstabiliser le gouvernement congolais en place. Lorsqu'il revint au pays, il rallia la jeunesse à sa cause et organisa l'insurrection populaire qui éclata en 1963. ${ }^{26}$ Pour Mulele, il existait au Congo deux classes sociales depuis le temps colonial : les riches et les pauvres; les oppresseurs et les opprimés. Ainsi, tous les moyens de production étaient pratiquement contrôlés par la première classe qui pouvait ainsi pérenniser sa position au détriment de la masse populaire qui se retrouvait dans une extrême pauvreté. ${ }^{27}$ S'appuyant sur ces convictions, ${ }^{28}$ Mulele chercha à obtenir « la deuxième indépendance » en vue d'instaurer le socialisme. Le Kwilu ${ }^{29}$ se retrouvait en première

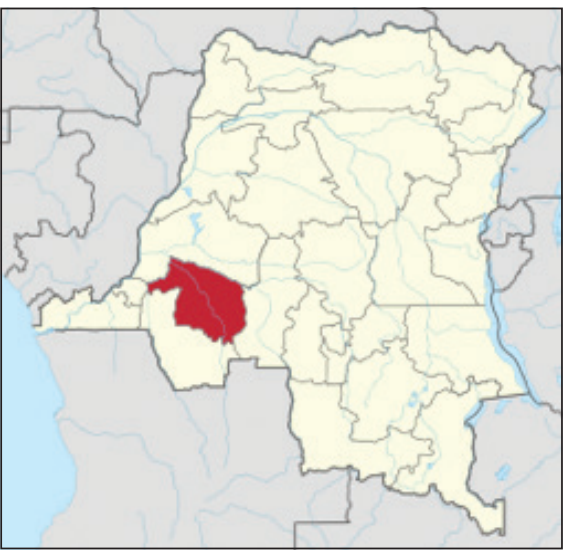

Figure 1. Carte du Congo ${ }^{30}$ ligne et au cœur même de la rébellion, de par sa position géographique.

Indifféremment appelé rébellion $d u$ Kwilu ou de Mulele, le mouvement séditieux se répandit comme une traînée de poudre dans les trois quarts du territoire national congolais, jusqu'à la province orientale et même dans celle du Kivu, frontalière du Rwanda. À Kikwit, les ecclésiastiques étaient la cible des rebelles, car selon la pensée de Mulele et de ses partisans, ils faisaient partie de la classe des nantis. Ainsi se développa une véritable haine envers les missionnaires et leurs œuvres apostoliques. Le discours prononcé par une religieuse missionnaire à l'occasion du jubilé d'or de la présence de Sœurs Annonciades ${ }^{31}$ résume bien l'état d'âme de ces religieuses, 18 ans après les troubles mulelistes :

La rébellion avait semé la destruction de nos postes : Kikombo n'était que ruines, Totshi et Gungu avaient l'aspect d'un paysage anéanti par des bombardements [...]. La plupart d'entre nous se rappelleront pour toujours ces 
sombres années jalonnant non seulement la perte de nos œuvres apostoliques, mais surtout la méconnaissance des sacrifices consentis par nos missionnaires en faveur de ce peuple. Oui beaucoup de nos filles en pâtirent également. Paix aux âmes de celles qui en ont succombé! ${ }^{32}$

Quant à la réalité scolaire, les écoles furent fermées, les élèves, les enseignants, les populations entières souffrirent de nombreux maux. ${ }^{33}$ Les enfants étaient les plus à plaindre.

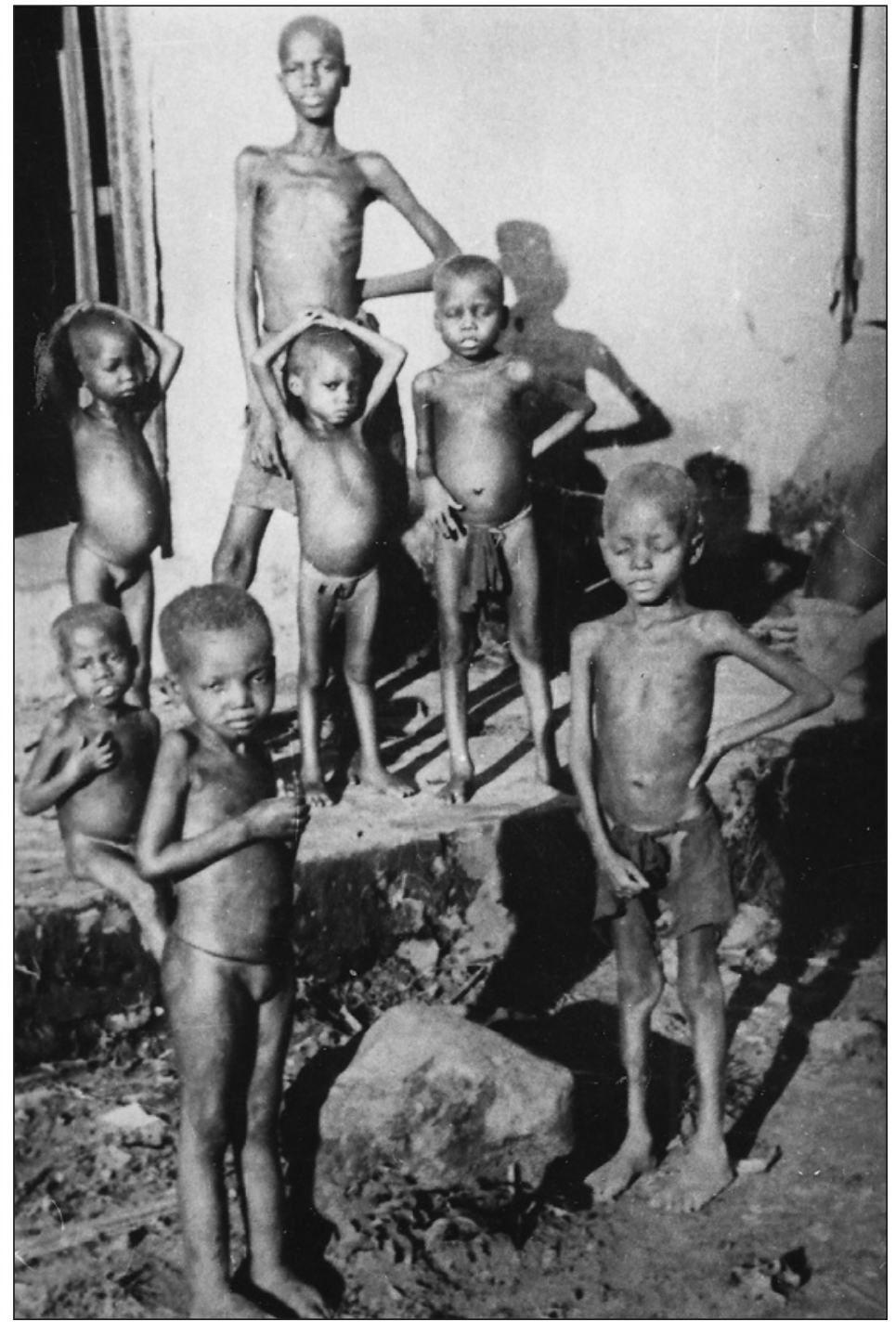

Figure 2. Enfants de Kikombo après la rébellion muleliste, $1965 .^{34}$ 
La rébellion a eu un impact particulièrement funeste sur les écoles de filles. À l'Institut Saint-André de Kikwit, ${ }^{35}$ les sœurs gardaient les élèves au pensionnat pendant toute la période de l'insurrection populaire. Les religieuses et les élèves vivaient dans la crainte permanente de subir des atrocités physiques et morales de la part des rebelles s'étant établis dans la forêt à proximité. Et même si les rebelles n'ont pas détruit cette école, ils s'en prirent tout de même aux religieuses. Un des maquisards se rappelle :

J'étais à Kikwit Sacré-Cour, nous étions installés dans la forêt derrière l'Institut Saint-André. Un soir, nous sommes allés chez les sœurs pour demander la nourriture. En sortant de chez elles, nous les avons frappées. Deux sœurs ont été grièvement blessées : Anne-Marie Billiet et Marie-Françoise Lowie. Je les connaissais bien parce que j'étais du makwela ${ }^{36}$ et mon père travaillait à la mission. ${ }^{37}$

À la mission Totshi, ${ }^{38}$ les rebelles installèrent leur maquis derrière l'école primaire des filles qu'ils saccagèrent à leur gré. Aussi paradoxal que cela puisse paraître, le témoignage de l'abbé Kwambika ${ }^{39}$ aux autorités politiques souligne que le déchaînement de la rébellion fut causé non seulement par les rebelles, mais aussi par les autorités administratives : «Mauvais traitements infligés à la masse : hommes, femmes, enfants. Viols des femmes et des filles. Les hommes devaient parfois fermer les yeux pour ne pas tout voir [...]. $»^{40}$ Dans ce poste, les religieuses furent encerclées par une brigade de rebelles qui les harcelaient quotidiennement. Dans une lettre du 28 juin 1964, le curé de la mission catholique de Totshi écrivait : "J'ai été à Totshi. Les sœurs sont vraiment entourées de rebelles. Cela n'empêche qu'ils ne font qu'ennuyer les sœurs : 11 perquisitions en un jour. Et du matin au soir il y a toujours une nouvelle bande pour demander des médicaments [...]. Les unes sont favorables, les autres veulent à tout prix tuer les sœurs. Qui gagnera ? ". ${ }^{41}$

La situation d'insécurité était similaire à Kisandjii ${ }^{42}$ qui perdit son école d'apprentissage pédagogique. Certaines filles en ont gardé des souvenirs indélébiles. Les récits poignants de quelques maquisardes traduisent clairement le caractère tragique de la rébellion. Interrogée sur la situation lors des émeutes, C.K. répondra :

J'étais en $1^{\text {ère }}$ année de l'école d'apprentissage pédagogique lorsque la rébellion a commencé à la mission Kisandji. Toutes mes amies étaient parties, il m’était impossible d'atteindre le village vu le danger que j'encourais. Je suis restée au dortoir avec quelques condisciples. Un soir, nous avons entendu des bruits. C'étaient des rebelles qui pillaient la maison des sœurs. Nous regardions de loin comment les sœurs étaient maltraitées. Lorsque les rebelles sont arrivés chez nous au dortoir, la scène était horrible à voir : ils nous rouaient de coups et violaient les élèves l'une après l'autre. Celles qui résistaient étaient tuées surle-champ. Une des religieuses en était devenue folle... J'étais obligée de partir de la mission et de rejoindre ma famille pour échapper au sort de nombreuses jeunes filles. Mais en cours de route, j'ai été rattrapée par les rebelles qui m’ont emmené au maquis. ${ }^{43}$ 
Ce témoignage semble être confirmé par celui d'une autre maquisarde :

Depuis ma puberté jusqu'aujourd'hui, ma vie est toujours marquée par le souvenir indélébile de la rébellion. C'était très pénible de vivre cette période de troubles. Il y avait des victimes ensanglantées par-ci par-là. ${ }^{44}$

De toute évidence, l'analyse de tous ces entretiens révèle plusieurs facettes des perturbations psychiques vécues au cours de cette période confuse. En plus, on ne peut nier que sur le plan de l'éducation nationale, la rébellion a aussi entrainé d'autres conséquences : "le système éducatif du Congo a été cassé par l'exécution délibérée et systématique des instituteurs par les rebelles [...], les élites moyennes ont été décimées. Les molakisi des écoles avaient été délibérément sacrifiés [...]. " ${ }^{45} \mathrm{Il}$ est évident que l'image que nous venons de donner de la rébellion relève un aspect tragique des circonstances. En effet, beaucoup d'auteurs ${ }^{46}$ qui se sont penchés sur l'histoire de la rébellion muleliste ont davantage souligné le caractère dramatique des événements. Cependant, le fait de s'attarder sur les détails de cette révolution populaire peut aussi biaiser la réalité. Comme nous l'avons souligné, il n'est jamais possible-en dépit du fait que nous tenons compte à la fois de l'histoire des révolutionnaires et de celle des victimes — d'éliminer l'élément subjectif du récit historique, puisqu'il est quand même toujours rapporté selon un certain point de vue. ${ }^{47}$ Dans cette perspective, nous ne pouvons manquer de relever les valeurs pédagogiques des écoles de Mulele installées dans les différents maquis. Sur les 32 maquisards interviewés, 26 (soit 81,2 \%) étaient en faveur de la rébellion, malgré la terreur qu'elle engendrait au quotidien. Pour eux, la révolution populaire n'était pas une explosion de sauvagerie, mais plutôt une expression d'insatisfaction face à l'euphorie de l'indépendance, qui s'était avérée illusoire dès les premières années. On voit d'ailleurs ici se manifester un autre paradoxe intéressant sur lequel nous reviendrons plus tard : la question de savoir dans quelle mesure la rébellion a dévié dans la pratique des objectifs éducationnels et des intentions annoncés par Mulele. Il est donc grand temps de nous intéresser de plus près à son projet " pédagogique ".

\section{Vers une africanisation de l'éducation}

L'idéologie prônée dans les maquis mulelistes s’incarnait dans la "décolonisation mentale ", parce que "l'indépendance impliquait une indépendance politique et, dans une moindre mesure, économique, mais l'indépendance culturelle [...] s'avérait beaucoup plus difficile à réaliser $"{ }^{48}$ Ainsi, le projet éducatif muleliste était plus lié à la nature de l'éducation traditionnelle. Celle-ci visait à ce que l'enseignement soit compatible avec le vécu des apprenants. En effet, le reproche le plus fréquent par rapport à l'école congolaise, c'est d'avoir conservé les stigmates de la période coloniale. ${ }^{49}$ Mais il faut d'abord préciser le sens qu'il convient d'accorder à cette critique. Il convient de reconnaître que, dans l'école coloniale ou postcoloniale, l'enfant ne s'abreuve pas à ses sources premières, et peu de choses dans le système éducatif officiel lui suggèrent l'apprentissage de sa société et de sa culture. Cette dépersonnalisation l'entraine à 
assimiler une nouvelle culture dite "moderne " et à rejeter, par conséquent, tout héritage du passé. La seule préoccupation de l'enfant est de réussir à l'école, comme celui qui a été le premier dans son village à bénéficier de l'éducation occidentale. On assiste ainsi à une valorisation du travail intellectuel à l'instar de l'ancien colon belge, du premier intellectuel de son village; mais on a aussi simultanément une perception dégradante du travail de la terre tel que valorisé par l'école ancestrale.

Ainsi, on considère la conquête de l'Afrique par les Européens comme une désintégration de la société traditionnelle considérée par les colonisateurs comme réservoir des antivaleurs. Mais le problème de l'éducation missionnaire était qu'elle n'était que fort peu axée sur l'émancipation de l'individu. Comme la recherche louvaniste a fini par démontrer, il est ici bien plus question de " paternalisme " que d' "émancipation " ${ }^{50}$ Non seulement l'espace mental que visait cette formation était beaucoup trop étroit pour mener à l'émancipation, mais le point de vue que les missionnaires adoptaient par rapport à la population autochtone témoignait en outre d'un trop grand paternalisme. Ils voulaient décider de ce qui était bon pour les Africains. $\mathrm{Ou}$ comme l'a déclaré Marvin Markowitz au cours de la première décennie suivant l'indépendance :

The Catholic and Protestant missionaries in the Congo had, through their presence and action transformed Congolese society. They helped them to cope with and adjust to colonial reality, and eventually, to oppose it. Yet the missionaries could not provide the focus for true national integration. Their failure, if one can call it that, was tinged with egotism and paternalism. They saw themselves in the platonic sense as leaders of colonial society, as permanent, rather than transitory, figures of authority to the Congolese. They believed that they could discern and control the direction of social and political development with only scant reference to the urban and rural masses. When this proved fallacious and disastrous they withdrew from direct political involvement. ${ }^{51}$

Ce manque d'émancipation explique le malaise et la confusion que ressentent les élèves pris entre deux cultures qui s'opposent totalement. Les conséquences apparaissent dans les comportements de beaucoup de jeunes, non seulement les soi-disant hommes "évolués ", ${ }^{52}$ mais plus particulièrement les filles de toutes les couches sociales, qui font preuve de tant de lacunes importantes dans plusieurs domaines de la vie. Elles témoignent d'un manque de repères sociaux parce que tout tend à la modernisation et valorise la culture des modèles copiés de l'étranger. Au fond, la situation des filles est restée similaire à celle des femmes dans l'ensemble de la société congolaise postcoloniale. En raison des us et coutumes, la femme est réduite au rôle de ménagère et de procréatrice. La maternité lui confere un statut de prestige et apparaît à la fois comme un état d'épanouissement de sa féminité. C'est pour cette raison que le mérite de la femme ne tient pas de sa personnalité ou de son avenir, mais plutôt du rôle qu'elle joue dans la procréation. On comprend alors que la scolarisation des filles soit perçue comme une activité contraire aux traditions et qui risque de réduire leurs capacités à procréer et de les en détourner pendant de longues années. Le rejet 
instinctif de la scolarisation des filles ${ }^{53}$ explique, pour une grande part, le nombre de femmes congolaises souffrant d'analphabétisme. ${ }^{54}$ Elles sont d'ailleurs surchargées par les tâches ménagères et le travail aux champs. Certaines recherches, comme celles de Kita, précisent même qu'à l'indépendance, aucune femme ne comptait parmi les centaines des diplômés congolais de l'enseignement secondaire ni, évidemment, parmi les quelques centaines d'étudiants fréquentant les institutions d'enseignement supérieur et universitaire. ${ }^{55}$ Effectivement, la quête d'émancipation de la femme fut un long cheminement depuis l'accession du pays à l'indépendance. Sans toutefois généraliser les faits, les disparités hommes-femmes en matière de fréquentation scolaire sont restées visibles sur l'échiquier national. Les statistiques du Bureau de l'enseignement catholique $^{56}$ des années 1962-1963 prouvent l'écart considérable entre le taux de scolarisation des garçons et celle des filles. Sur un total de 44846 élèves inscrits de la première année à la sixième année dans les écoles du réseau catholique, on trouve 35959 garçons (80,18\%) et 8887 filles (19,82\%). Il ne fait donc pas de doute qu'au moment de l'indépendance, le taux de scolarisation des filles était beaucoup faible que celui des garçons. Cependant, signalons qu'au Congo, le problème de l'éducation des filles se pose différemment dans les grandes villes que dans les villages. On ne trouve généralement pas les mêmes conceptions de l'école dans ces deux milieux. Dans les villes, le brassage des cultures modernes et traditionnelles permet aux filles de considérer l'école comme une des voies de promotion sociale. Elles y accèdent facilement malgré le coût élevé des frais scolaires. ${ }^{57}$ Dans les campagnes, certains villageois considérèrent plutôt que la promotion des filles via l'école représente une atteinte à l'autorité masculine. Cette conception traditionnelle qui ne prend pas en compte l'importance de la scolarisation des filles est restée prédominante et même omniprésente pendant des décennies. Durant les premières années d'indépendance du Congo, Pierre Mulele s'est rendu compte de ce problème, qui n'était qu'un des nombreux autres problèmes structurels auxquels il était confronté en tant que ministre de l'Enseignement. ${ }^{58}$

C'est pour cela qu'il a prôné une politique d'africanisation du système éducatif qui prit forme pendant la révolution populaire. En pratique, la philosophie de la décolonisation mentale s'incarnait sur la tradition ${ }^{59}$ dans laquelle la fille s'éduquait par le contact, par l'exemple, ainsi que par des ordres et des défenses. Comme dans l'éducation traditionnelle, Mulele visait « l'apprentissage technique, l'adaptation aux usages, aux coutumes, la révélation des croyances et du sens des rites, et finalement introduction dans le groupe des adultes par certaines cérémonies à caractère superstitieux $"{ }^{60}$ L'éducation maquisarde était informelle et les stratégies peu institutionnalisées. Il y avait des maîtres et des élèves, mais la différence était surtout visible au niveau des infrastructures scolaires et des méthodes d'enseignement. Il existait au total 21 maquis fonctionnant comme des écoles au sens large, parsemées dans toute la région du Kwilu. Les maquis étaient situés « dans une zone triangulaire sur le territoire de la tribu Bambunda : Lukamba-Yasalokwa-Imbongo. Et plus tard, ils furent installés sur toute l'étendue du territoire des tribus Bapende et Badinga ». ${ }^{61}$ Le premier maquis de Lukamba comptait déjà " au mois d'août 1963, 580 partisans, dont 150 jeunes filles ${ }^{62}$ Les écoles étaient placées en pleine forêt ou dans la brousse avec des moyens primitifs : quelques troncs d'arbres servant de bancs avec des feuilles 
disposées au-dessus pour abriter des intempéries; des lianes attachant les arbres les uns aux autres en signe de clôture afin de distinguer le lieu des enseignements appelé communément " Département de l'éducation »; des ardoises et des touches pour la prise des notes. Les classes étaient généralement mixtes, mais pour quelques leçons, la séparation des sexes était de rigueur. Le nombre des filles égalait parfois celui des garçons parce que " pour beaucoup de paysans, avoir un enfant au maquis constituait une fierté; par surcroît, la présence des filles exprimait à la fois le sens de l'endurance et de la lutte collective contre les inégalités sociales ${ }^{63}$

Les effectifs variaient, selon les personnes interviewées, entre 300 et 600 élèves répartis en cinq ou six classes par maquis (école). Dans la plupart des cas, les enseignants étaient des moniteurs ou des instituteurs de formation. Leur présence nombreuse dans le maquis se justifiait par une recherche accrue du bien-être non réalisé par le gouvernement en place. Les témoignages de cet enseignant en soulignent la pertinence :

Lors de leur campagne électorale, les membres du Parti socialiste africain (P.S.A.) nous avaient promis une vie plus agréable après l'indépendance. À nous les enseignants, il était question de tripler notre salaire. C'est alors que nous les avons votés. Ils ont tous occupé des fonctions importantes au sein du gouvernement. Les années passaient, notre situation ne s'améliorait pas. Ceux que nous avons élus s'enrichissaient davantage, mais nos enfants et nousmêmes croupissions dans la misère. C'est ainsi que j'ai décidé de suivre Mulele dans le maquis pour lutter contre ces inégalités intolérables. ${ }^{64}$

Un autre enseignant témoigne :

Une chose me tenait à cœur : faire la guerre pour déstabiliser ceux pour qui nous avons voté et qui ont oublié leurs promesses en nous laissant dans une pauvreté bien plus grande qu'avant l'indépendance. ${ }^{65}$

À cet égard, il n’est guère étonnant que les enseignants se soient représenté la nouvelle société postrévolutionnaire conçue par Mulele, comme un gigantesque village dans lequel les gens retrouveraient le bonheur. ${ }^{66} \mathrm{La}$ tradition commandait toute la philosophie éducative des maquisards. Aussi nous interrogeons-nous à savoir comment s'organisait l'éducation des filles dans les maquis et quelle était la nouveauté dans ce type d'éducation?

\section{L'éducation de la fille maquisarde}

Parmi les objectifs de toute éducation, on compte la socialisation et l'éclosion de la personnalité de l'individu. Ces objectifs n’ont pas été ignorés par Mulele. Schématiquement, la socialisation est l'étude génétique de trois aspects du développement : les relations interpersonnelles, les représentations sociales et les activités sociales ou socialisées. ${ }^{67} \mathrm{Il}$ ressort de cette conception de la socialisation que, dans toute 
société, la nécessité d'intégration adéquate de chaque membre s'avère un impératif. Ainsi, les filles maquisardes étaient appelées à vivre ensemble pour mieux s’imprégner de l'esprit de la décolonisation mentale. Le recrutement au maquis se faisait sans préparation préalable et ne se basait que sur un simple hasard. Par ailleurs, quelques filles parmi les répondantes ont reconnu s'être inscrites à l'école maquisarde parce qu'elles étaient convaincues que le maquis leur apporterait le bien-être individuel et collectif tel que Mulele leur promettait : "Les Noirs construiront eux-mêmes leurs usines et fabriqueront ce dont ils ont besoin. Les filles apprendront à fabriquer des assiettes, des verres, des casseroles $"{ }^{68}$

Au maquis, les journées scolaires commençaient à cinq heures du matin après le réveil brusque du sifflet du chef d'équipe. Puis suivaient des exercices physiques de nature militaire : marcher, courir, sauter, ramper et manier les armes. Outre les garçons, toutes les filles devaient s'entraîner pour combattre, repousser et vaincre les ennemis : "On ne pouvait distinguer les filles des garçons, nous étions tous habillés en pantalon pour grimper sur les arbres; sauter sans crainte de grands trous creusés en guise d'embuscades, se coucher par terre au surgissement des avions, attaquer les soldats. » ${ }^{69}$

L'exigence de l'école maquisarde consistait à former les combattants pour la révolution. Par les exercices physiques, les filles étaient éduquées à l'endurance. Pour les maquisardes elles-mêmes, l'éducation à l'endurance rejoignait les pratiques de l'initiation exercées dans leurs villages respectifs à l'âge de la puberté. De cette manière, l'action éducative de Mulele était fortement imprégnée de traditions culturelles. À titre illustratif, l'endurance apprise par l'entremise de « Giwila » du peuple Bapende, ou le « Malongi » des Bambala et le «Tshikumbi » ${ }^{70}$ chez les Batshokwe, aidait les filles à garder une attitude calme devant les contrariétés de la vie parce que culturellement, il était mal vu qu'une femme exprime ostensiblement ses besoins et ses désirs. Les rites et les méthodes divergeaient selon les ethnies, mais la conception de l'éducation s'appuyait sur le programme selon lequel les garçons commençaient à être intégrés dans l'intimité des hommes et les filles dans celle des femmes. Cependant, il faut préciser que l'éducation des garçons visait à leur enseigner leurs futures responsabilités en tant que chefs de famille, ou de guerriers chargés de la défense du village.

Toutes ces pratiques relevaient de la philosophie de l'école traditionnelle. Nous sommes ainsi frappés par la similitude avec l'éducation maquisarde qui, à travers ses exercices physiques, inculquait l'endurance aux filles comme symbole culturel de base. Dans cette optique, ce genre d'éducation scolaire ne pouvait que les inciter à demeurer dans le maquis. Ce qu'exprimait une maquisarde avec enthousiasme : "Souffrir de la faim, parcourir les forêts et les brousses en pleine nuit, affronter les dangers des animaux sauvages, marcher sous les pluies torrentielles, attaquer les ennemis [...] était notre lot quotidien et constituait notre fierté ». ${ }^{71}$

Outre de la fierté, les exercices physiques revêtaient pour les filles, une valeur thérapeutique : celle de vaincre la peur. Dans la nouvelle société conçue par Pierre Mulele comme une conquête de la deuxième indépendance, la femme devrait être l'égale de l'homme. Cet extrait des exhortations qu'il adressait aux femmes dans le maquis de Mulemba le confirme avec pertinence : 
Il y a des pays où les femmes ont lutté aux côtés des hommes. Angela Davis est une Afro-américaine qui a beaucoup lutté. Valentina Terescova a été la première astronaute. Vous voyez ce que les femmes peuvent faire dans les pays socialistes. En Chine, $j$ 'ai vu les femmes travailler comme ingénieur, directeur d'entreprise, $j$ 'en ai vu commander dans l'armée, conduire les chars. ${ }^{72}$

Le courage et la bravoure étaient non seulement la voie de l'émancipation des filles, mais surtout une des principales caractéristiques de l'action éducative. Pour y parvenir, les enseignants utilisaient la drogue pour combattre la peur féminine : «Dans le maquis, nous étions toutes de petites toxicomanes. Il le fallait bien pour tenir le coup et par surcroît, procéder le cas échéant aux pratiques d'assassinat $» .{ }^{73}$

Le courage remarquable des filles s'est exprimé dans le maquis de Mulemba, près d'Idiofa lors du jugement et de l'exécution de l'abbé Lakwan, prêtre partisan de la rébellion considéré comme traître et dont le réquisitoire a été prononcé par une maquisarde. Pour de nombreuses filles, cet exemple a suscité leur motivation de "parfaire leur éducation et atteindre l'égalité avec les hommes $»{ }^{74}$ Le programme d'enseignement se bornait aux leçons de politique, de religion, de géographie et de langue. À cela, s'ajoutaient des rudiments de savoirs traditionnels.

La leçon de politique appelée communément «malongi ya politique »" (en langue Kikongo) par les maquisards, leur fournissait une forte préparation idéologique au marxisme et au socialisme. Les idées véhiculées dans ce cours se rejoignent dans les réponses des interviewés :

Pendant le "malongi ya politique ", nous apprenions les noms des hommes célèbres comme Mao, Marx, Lénine ou Staline qui ont lutté contre les inégalités sociales à travers les révolutions. Les maîtres nous enseignaient aussi les noms des pays socialistes : la Chine, la Russie, l'Albanie et l'Union soviétique. Dans ces pays, tout le monde avait les mêmes chances. Comme partisans de $\mathrm{Nga} \mathrm{Maan}{ }^{76}$ nous aspirions tous à ce genre de société. ${ }^{77}$

Une autre maquisarde s'explique en ces termes :

Les moniteurs s'amenaient toujours avec un petit livre de $\mathrm{Mao}^{78}$ auquel ils y recouraient constamment sous les tentes du maquis. Ils insistaient beaucoup sur le combat contre les inégalités sociales. Notre objectif était d'arracher la deuxième indépendance du Congo. Nous vivions dans une grande misère, mais à côté de nous, les nantis s'enrichissaient davantage. Notre lutte était collective : se retrousser les manches et travailler la terre que nous avaient léguée nos ancêtres. C'est par elle que nous pourrions être sauvés et vivre comme eux sans attendre l'aide extérieure qui supprimait notre dignité en nous rendant quémandeurs à vie. ${ }^{79}$

Il y a lieu de retenir ici deux enseignements : la valeur de la terre comme héritage ancestral et l'importance du travail (surtout le travail manuel). En combinant ces 
deux enseignements, l'idée sous-jacente de l'éducation des filles selon Mulele était entre autres de combattre l'oisiveté et de préserver l'attrait au travail. Dans cette perspective, l'école maquisarde voyait dans l'activité manuelle un moyen de formation du caractère des filles. C'est dans ce cadre qu'elle leur inculquait l'activité manuelle dans un souci de développement mental et collectif. En plus, la formation de la fille était toujours axée sur les vertus sociales et le sens patriotique. ${ }^{80}$ Car, " les valeurs ne s'apprennent jamais dans des cours spécifiques qui peuvent tout au plus, dans ce domaine, prétendre à une information des élèves. Elles se construisent dans l'ensemble des situations éducatives et des séquences d'enseignement si celles-ci sont capables de montrer que l'on apprend mieux dans la coopération " ${ }^{81}$ À travers les interdits et les tabous, on apprenait également à la fille maquisarde ce qu'elle doit faire et ce qu'elle doit éviter au risque d'être frappée par le mauvais sort. Pour les rebelles mulelistes, les ancêtres veillaient sur leur vie et y intervenaient de temps en temps. Aussi était-il impérieux pour la fille maquisarde de connaître les ancêtres de son clan dont elle respectait les interdits, car d'eux dont dépendait aussi la santé et la prospérité. Conjointement, le but ultime de l'éducation des filles consistait à les pourvoir des connaissances indispensables et essentielles à l'existence, et surtout de les guider dans l'acquisition des vertus morales et des autres expériences de la vie conjugale. C'est dans ce cadre que les filles étaient informées sur certaines questions relatives à l'éducation sexuelle.

Les maquisards recevaient des tâches journalières : chasse, creusage des fosses, fabrication de "pupus $»^{82}$ et d'explosifs pour les garçons; pêche, ménage, ramassage du bois et cueillette de champignons pour les filles. Les après-midis étaient généralement réservés aux pratiques d'espionnage, aux audiences avec le chef d'équipe et à la résolution des problèmes journaliers. Le soir, les garçons se retrouvaient ensemble dans leurs bivouacs. Quant aux filles réunies autour de leurs éducatrices traditionnelles, elles apprenaient quelques strophes en guise d'éveil à la conscience révolutionnaire. Étant donné que la tradition ancestrale accorde une place essentielle à la chanson dans la transmission des enseignements, l'école maquisarde y recourait aussi dans la mesure où les patronnes chantaient ce qu'elles ne pouvaient pas dire aux filles. C'est en ce sens que l'apprentissage de certaines chansons, même " révolutionnaires ", revêtait une valeur culturelle. En même temps, la parole restait le support prioritaire dans la mesure où « elle en exprimait le patrimoine traditionnel [...], elle tissait entre les générations passées et présentes ce lien de continuité et de solidarité sans lequel il n'existe ni histoire, ni civilisation $"{ }^{83}$ Pour ce faire, les instituteurs exploitaient la communication orale pour transmettre le savoir éducatif. L'avantage réside dans le fait que les filles pouvaient s'exprimer librement à l'opposé des tendances éducatives de l'école congolaise où prédominent les interdictions et les menaces de sanctions. Cette approche de la pédagogie de Mulele confere à son école une dimension africaine : la parole reste la principale expression de la pensée humaine, l'écriture n'en constitue qu'un support qui ne lui ôte en rien son importance immuable. À travers les contes, les proverbes et les maximes, la matrone transmet aux filles les normes destinées à les guider dans leur accomplissement humain. Ainsi, l'éducation s'organisait « autour de quelques grands thèmes, de quelques images et symboles très concrets servant de cadre, de catégories et 
de structures à la pensée $" .{ }^{84}$ Ce genre d'enseignement avait souvent lieu le soir au clair de lune pour symboliser la communion avec les ancêtres et les autres êtres invisibles qui assuraient la protection des maquisards. Vue sous cet angle, l'éducation maquisarde ne visait pas seulement un savoir ou une compétence, mais aussi toute la personnalité des matrones qui aidaient les filles dans les différents domaines de la vie. Ainsi, il se développait chez les matrones une attitude de responsabilité consentie envers les filles. Elles devenaient davantage des accompagnatrices que de simples institutrices dont l'instruction prévalait sur l'éducation : "La parole se faisait sur le mode de la discussion et de l'explication, et non plus sur celui de l'imposition ». ${ }^{85}$ Il en résultait pour les filles le sentiment de responsabilité dans l'édification de la nouvelle société.

Pour bien mener la révolution, les maquisards étaient appelés à s'imprégner de l'esprit du groupe. C'est pour cette raison que l'éducation des filles accordait une importance à la cohésion conformément aux principes majeurs de la doctrine muleliste : "Nous voulons que tous les hommes soient heureux. L'homme qui abandonne le parti du peuple mourra de la même manière que le poisson qui abandonne l'eau ». ${ }^{86}$ Dans cette perspective, une invitation à l'unité était adressée à tous les maquisards. Voici un extrait de la lettre de Mulele, écrite à ses compagnons de lutte, qui illustre bien cette pensée : "Camarades, je vous prie de lancer un appel à l'unité [...]. Je vous demande d'oublier vos querelles stériles du passé, de bannir l'esprit séparatiste. L'union devra reposer sur une doctrine de base conséquente, conforme à la ligne générale de la Révolution sur toute l'étendue du territoire congolais ». ${ }^{87}$

En ce sens, l'éducation des filles s'attelait à garantir non seulement l'esprit du groupe, mais aussi veiller à sa protection. Lorsque les conflits naissaient dans leur communauté, elles recouraient à la réconciliation autour d'un verre de vin de palme et la noix de cola en s'adressant aux ancêtres. Nous retrouvons les mêmes pensées dans la société traditionnelle où l'unité et la solidarité jouaient un rôle considérable dans la vie commune. Le contraire était vu comme un frein au développement de la société.

Dans le maquis, les enseignements se donnaient en langue locale. Ainsi, l'unité des maquisards était maintenue par la langue commune du milieu et de toute la région, le "Kikongo ", une des quatre langues nationales du pays. ${ }^{88}$ À cela s'ajoutaient quelques dialectes comme le Kipende, le Kibunda, le Kitshokwe, selon le lieu du maquis et la circonscription linguistique.

Il faut noter que depuis 1962, l'usage du français — qui est alors devenu la langue officielle de l'enseignement — entraîna différentes conséquences. Pour les élèves issus des familles nanties, la langue française ne pose presque pas de problème parce qu'ils l'ont acquise dans leur famille comme première langue de communication dès leur jeune âge. En revanche, ceux des écoles rurales dont la pratique orale du français se limite à l'école sont parfois conduits à des échecs scolaires à cause de leurs lacunes dans la langue de Voltaire. À ce titre, l'usage de la langue locale et des dialectes au maquis, résolvait le problème posé par le français, langue étrangère choisie par l'école coloniale et postcoloniale comme langue de l'enseignement. On pourrait imaginer que Mulele considérait l'usage du dialecte dans l'enseignement comme une expression de l'identité congolaise. Par conséquent, nous pouvons estimer que sa philosophie est révélatrice des valeurs pédagogiques. Certains cours, comme la géographie et 
l'histoire, suivaient le même processus en s'appuyant sur la maitrise du pays comme lieu essentiel d'environnement de l'élève congolais. Un ancien maquisard le souligne bien clairement : "Grâce au cours de géographie, j’ai renforcé mon sens patriotique : par des enseignements, j’ai appris à connaître les richesses de notre contrée : les rivières, les plateaux, les forêts et les vallées fertiles ". ${ }^{89}$

À l'école congolaise par contre, malgré la réforme du programme scolaire de 1961, le cours de géographie a continué de s'attarder sur la connaissance physique et économique des pays étrangers. Quant à celle du Congo même, elle reste plus au niveau de l'abstraction que de la concrétisation. Ce paradoxe s'expliquerait par le fait que le programme national n'a pas suffisamment pris en compte l'importance, pour un élève congolais, de connaître son milieu naturel. Il en est de même pour le programme d'histoire, encore très inspiré par l'éducation occidentale. La nouveauté de l'école maquisarde était de rapprocher les enseignements du milieu concret des apprenants, à la différence de l'école postcoloniale privilégiant le système de "cours magistral " et l'acquisition de connaissances étrangères à la vie des élèves. C'est que lorsqu'on maîtrise un milieu, on arrive à l'aimer. Il en va de même pour le pays : "lorsqu'on ne connaît pas un pays, on ne peut l'aimer, ni le bâtir, ni non plus le défendre $» .{ }^{90}$ C'est à ce titre que, contrairement à l'enseignement néocolonial, le sens de la guérilla se justifie dans les objectifs assignés non seulement pour le cours de géographie, mais aussi à l'ensemble de l'éducation maquisarde.

\section{Conclusion}

Comment peut-on finalement répondre à la question rhétorique que nous posions dans le titre de notre article? Comme c'est généralement le cas avec ce genre d'antithèses — dans le cas qui nous occupe, des jeux de mots fondés sur l'annonce proche du slogan du général Olenga par laquelle nous avons commencé-, la réponse est infiniment plus complexe et nuancée que ce que l'opposition énoncée laisse supposer. C'est pourquoi nous avons cherché dans ce qui précède à mieux comprendre ces complexités en les contextualisant dans le temps et l'espace. Car la recherche historique a le pouvoir de démontrer que la dichotomie n’a en soi que rarement été un bon point de départ pour le progrès social en Afrique. ${ }^{91}$ La réalité quotidienne prend réellement en compte l'hybridité historique développée en termes d'éducation, ${ }^{92}$ d'où notre tentative de valoriser les différents modèles éducatifs qui étaient en jeu.

Notre but ne consiste pas à idéaliser l'éducation traditionnelle, mais plutôt à identifier, par le biais de la rébellion, comment Mulele a pu résoudre en partie le problème d'incohérence des systèmes éducatifs moderne et traditionnel. Si toute éducation se nourrit des traditions et des valeurs de la société, elle contribue aussi à la création et au développement de nouvelles attitudes porteuses d'avenir. L'école traditionnelle renferme des valeurs importantes qu'il importe non seulement d'identifier, mais aussi de retenir et de préserver lorsqu'elles peuvent être utilisées dans les conditions actuelles. C'est dans cette optique que nous avons voulu relever les valeurs culturelles mises en place à l'école muleliste. Bien que la rébellion présente le caractère violent des événements, on ne peut non plus négliger le côté positif d'une éducation 
cohérente avec la culture africaine. L'école muleliste a valorisé, par exemple, des techniques efficaces et intéressantes de transmission d'acquisition de connaissances, telles que les contes et les proverbes. Ces techniques gagneraient à être exploitées aussi par l'école moderne afin de rendre l'action éducative plus efficace. Cependant, certaines valeurs de l'éducation moderne, telles que l'objectivité scientifique et l'ouverture au monde, pourraient aussi apporter une plus-value à l'éducation maquisarde, soit pour la parfaire et ainsi la développer, soit pour rectifier certains de ses effets néfastes.

Dans la jungle de la révolution, Mulele a su organiser un système éducatif qui a libéré les filles congolaises de l'emprise coloniale. Si l'objectif de l'éducation des filles en Afrique vise à aboutir à leur libération, la prise en compte du contexte culturel ne peut être négligée. Mulele s'était rendu compte de cette réalité, et a mis sur pied une action éducative propre à la culture africaine. C'est ainsi que les maquisardes sont parvenues à changer leur comportement et à revoir leur mentalité. L'école coloniale et postcoloniale par contre, n'a pas pu exploiter les valeurs ancestrales au service de la modernité. Cette attitude aurait permis la synthèse entre l'éducation moderne et traditionnelle; elle aurait enlevé à l'école moderne l'image d'une structure étrangère, imposée à des filles se sentant parfois exilées de leur culture, et donc de leur monde. En revanche, exclure tout ce qui vient de l'Europe dans le système scolaire conçu par Mulele est un préjudice, car l'école (en temps de paix ou guerre) est le chemin obligé vers la civilisation de l'universel, celle du rendez-vous du donner et du recevoir ${ }^{93}$ qui « oblige l'Afrique à acquérir la connaissance indispensable à tout dialogue avec les autres continents ". ${ }^{94}$ L'enjeu essentiel est donc de s'ouvrir aux apports culturels étrangers et aux contraintes de la technicité en vue d'une synthèse harmonieuse. Mais la question se pose : dans quelle mesure ce rêve souvent explicité dans des traités et des projets pédagogiques est-il réalisable dans une société postcoloniale si complexe, de par ses réalités historiques. Indépendamment de la question financière, dans un pays qui investit à peine dans le secteur de l'éducation, ${ }^{95}$ le passé pédagogique montre que, vouloir imposer une émancipation effective par des tendances dictatoriales est une tâche impossible, aussi bien dans le Nord que dans le Sud. ${ }^{96}$ Les dirigeants africains et les Congolais spécialement, ne s'en rendent pas encore compte...

\section{Notes}

1 Nicolas Olenga, "La décolonisation de l'esprit ", Révolution Congolaise, ${ }^{\circ} 1$ (2003). Cette devise nous vient du général Nicolas Olenga, à qui nous donnerons encore la parole ailleurs dans cet article. Mais revenons-en à ce premier numéro de Révolution Congolaise, qui cherchait non seulement à mettre en évidence le lien entre les doctrines de Lumumba et Mulele, mais aussi à le prolonger jusqu’à Kabila. Après la mort de Lumumba, Laurent Kabila a en effet rapidement rejoint les rebelles de Mulele. Il en est même devenu le ministre des Affaires étrangères. Voir Kris Berwouts, Congo's gewelddadige vrede (Berchem : Epo, 2017), 35-36; et Colette Braeckman, L'enjeu congolais. L'Afrique centrale après Mobutu (Paris : Fayard, 1999), 95 : «L'un des premiers gestes de Kabila après son arrivée à Kinshasa sera de rendre un solennel hommage au martyr de la révolution (Mulele). Une cérémonie se déroule le 3 octobre 1997 à Kinshasa, en présence de Léonie Abo, l'épouse de Mulele, et de quelques-uns de ses partisans ". 
2 Annette Lembagusala Kikumbi, L'éducation des filles dans les écoles secondaires du diocèse de Kikwit en R.D. Congo (1960-1997) (Leuven : KU Leuven, Faculté de Psychologie, thèse de doctorat, 2017), 269.

3 Marc Depaepe, «Writing Histories of Congolese Colonial and Post-Colonial Education : A Historiographical View from Belgium ", dans Bernita Bagchi, Eckhardt Fuchs et Kate Rousmaniere (eds.), Connecting Histories of Education. Transnational and Cross-Cultural Exchanges in (Post)Colonial Education (New York/Oxford : Berghahn Books, 2014), 41-60.

4 Voir, par exemple, Hein Vanhee et Geert Castryck, «Inleiding: Belgische historiografie en verbeelding over het koloniale verleden ", dans Hein Vanhee, Geert Castryck et Luc François (eds.), Afrikaanse geschiedenis - Belgische traditie, numéro spécial de la Belgisch Tijdschrift voor Nieuwste Geschiedenis 32, 3-4 (2002) : 305-320.

5 L'histoire de l'éducation des filles a reçu une attention particulière pour la première fois, en littérature historico-pédagogique dans le cadre d'un projet scientifique à Louvain, par Pierre Kita Masandi, "L'Éducation féminine au Congo belge ", Paedagogica Historica 40, 4 (2004) : 479-508.

6 Isidore Ndaywel è Nziem, "L'historiographie congolaise. Un essai de bilan ", Civilisations. Revue internationale d'anthropologie et de sciences humaines 54, 1-2 (2006), 248.

7 La collaboration avec le susnommé Pierre Kita Kyankenge Masandi a donné lieu à différentes études, notamment sur l'histoire des manuels et des chansons scolaires, voir par exemple : Marc Depaepe, Jan Briffaerts, Pierre Kita Kyankenge Masandi et Honoré Vinck, Manuels et chansons scolaires au Congo Belge (Leuven : Leuven University Press, 2003).

8 Marc Depaepe et Lies Van Rompaey, In het teken van de bevoogding. De educatieve actie in Belgisch-Kongo (1908-1960) (Leuven/Apeldoorn : Garant, 1995).

9 C'est là bien entendu un problème systématique, indépendant de l'historiographie (post)coloniale. Voir Marc Depaepe et Frank Simon, "Sources in the Making of Histories of Education: Proofs, Arguments, and Other Forms of Reasoning from the Historian's Workplace ", dans Paul Smeyers et Marc Depaepe (eds.), Educational Research: Proofs, Arguments, and Other Reasonings (Dordrecht: Springer, 2009), 23-39.

10 Voir Alf Lüdtke, The history of everyday life. Reconstructing historical experiences and ways of life (Princeton : University Press, 1995); Lisa Cary, « Unespected stories. Life history and the limits of representation ", Qualitative inquiry 5, 3 (1999) : 411-427; Philip Gardner, "Oral History in Education : Teacher's Memory and Teachers History ", History of Education Society 32, 2 (2003) : 175-188.

11 Voir Bogumil Jewsewicki, " De la vérité de mémoire à la réconciliation. Comment travaille le souvenir? ", Le débat 5, 122 (2002) : 63-77; Bogumil Jewsewicki, Récits de vie et mémoires : vers une anthropologie historique du souvenir (Québec/Paris : Safi/ L'Harmattan, 1985).

12 Pour nos recherches sur les expériences scolaires, nous avons notamment demandé la collaboration des anthropologues Filip De Boeck et Césarine Bolya afin qu'ils interrogent pour nous quelques acteurs incontournables à Kinshasa, voir : Honoré Vinck, Jan Briffaerts, Frederik Herman et Marc Depaepe, « Expériences scolaires au Congo belge. Étude exploratoire ", Annales Aequatoria 27 (2006) : 5-101.

13 Les archives des Sœurs Annonciades d'Heverlee (Leuven en Belgique), qui étaient actives dans le domaine de l'éducation des filles au Kwilu, l'épicentre de la révolte de Mulele. Voir Ria Christens, Terra Incognita. 75 jaar annuntiaten in Afrika (Heverlee: Annuntiaten, 2006).

14 Carnet de notes tenu par un chef d'équipe des maquisards, rencontré à Kikwit, dans sa documentation personnelle. 
15 Dans bien des cas, la réalité de la rébellion a été réellement vécue par les maquisards. Cependant, elle porte parfois un caractère subjectif. Nous estimons que les faits racontés ne sont pas nécessairement une réalité, mais plutôt des souvenirs que nous pouvons considérer, dans certains cas, comme acceptables ou pas.

16 Ces cinq questions principales étaient : 1-Quelles étaient les raisons fondées de la rébellion muleliste?; 2-Dans quel maquis étiez-vous pendant la période de la révolution?; 3-Comment s'organisaient les journées?; 4-Que retenez-vous du temps passé au maquis?; 5-Quelles sont les conséquences de la rébellion sur l'éducation des filles?

17 Marc Depaepe, "Colonial Education in the Congo - a question of "uncritical" pedagogy until the bitter end? Encounters ", Encounters on Theory and History of Education 18 (2017) : 2-26.

18 Heinz-ElmarTenorth, "Lob des Handwerks, Kritik der Theorie - Zur Lage der pädagogischen Historiographie in Deutschland ", Paedagogica Historica 32, 2 (1996) : 479-508.

19 Benoit Verhaegen, Mulele et la révolution populaire au Kwilu (Paris : L'Harmattan, 2006).

20 Ludo Martens, Une femme du Congo (Bruxelles: EPO, 1991).

21 Renée Fox, Willy De Craemer et Jean-Marie Ribaucourt, « La Deuxième indépendance : Étude d'un cas. La rébellion du Kwilu ", Études congolaises 8, 1 (1965) : 1-35.

22 Voir Émile Janssens, Un peu de clarté : documents à propos de la décolonisation du Congo belge (Bruxelles : Mimeo, 1987); Thomas Kanza, The Rise and Fall of Patrice Lumumba: Conflict in the Congo (London : Peguin Books, 1979) ; Luc De Vos, Emmanuel Gerard, Philippe Raxhon, Jules Gérard-Libois, Lumumba. De Complotten? De Moord (Leuven : Davidsfonds, 2004), 634.

23 Olenga, "La décolonisation de l'esprit » 5.

24 Natif de l'ethnie des Bambunda le 11 août 1929 dans la localité de Nkulu Matende (situé à quelque $200 \mathrm{~km}$ à l'est de Kikwit au territoire de Gungu), Pierre Mulele dont le mouvement porte son nom mourut assassiné à l'âge de 39 ans dans la ville de Kinshasa. Originaire de Lukamba, village situé au nord de la paroisse de Totshi, il initia la rébellion sanglante qui éclata en 1963 dans le Kwilu au Congo.

25 Olenga, "La décolonisation de l'esprit » 6.

26 Georges Nzongola-Ntalaja, The Congo from Leopold to Kabila : a People's History (London/New York : Zed Books, 2007), 128-131.

27 Voir Martens, Une femme du Congo; Jean-Marie Ribaucourt, Évêque d'une transition René Toussaint 1920-1993. Missionnaire au Congo-Zaïre (Kinshasa : Baobab, 1997) ; Verhaegen, Mulele et la révolution populaire au Kwilu.

28 Mulele était décrit par $\mathrm{M}^{\mathrm{gr}}$ Gillon, ancien recteur de Lovanium (l'université catholique de Kinshasa), comme un homme hautain, anticlérical, fanatique, partisan (tout cela en contraste avec Lumumba). Voir Luc Gillon, Servir en actes et en vérité (Paris/Gembloux : Duculot, 1988), 162-164.

29 Précisons tout de même que le Kwilu se situe au Congo dans la province de Bandundu. Au cours des années 1960, il était un district et regroupait cinq territoires : Gungu, Idiofa, Masi-manimba, Bulungu et Bagata. Aux termes de la récente réforme territoriale décrétée en 2015, le Kwilu est devenu une entité provinciale à part entière.

30 La partie localisée représente la superficie actuelle du Kwilu sur l'échiquier national. Source : Wikipédia, Province du Kwilu, [en ligne], URL : https://fr.wikipedia.org/wiki/ Kwilu, page consultée le 26 avril 2017.

31 Congrégation missionnaire d'origine belge résidant dans le Kwilu depuis 1931. Elles furent les premières religieuses à s'installer dans la partie sud à la paroisse de Kikombo. Le lycée de filles qu'elles créèrent dès leur arrivée en ce lieu fut complètement détruit pendant la révolution populaire. 
32 Archives des Annonciades d'Heverlee, Correspondances missionnaires : 8.2.4.5/1. Discours du jubilé d'or, Kingandu 25 mars 1981.

33 Ria Christens, Terra Incognita, 111-114.

34 Source : Cultureel Erfgoed Annuntiaten Heverlee. Audiovisual Fund Picture n ${ }^{\circ}$ AVA A 3322.

35 Comme le nom l'indique, cette école est une œuvre des religieuses de Saint André dont la création remonte en 1952.

36 Quartier des gens mariés composé des enseignants, infirmiers ou travailleurs dans les paroisses catholiques.

37 Témoin $n^{\circ} 17$, G. M. Kikwit, 8 décembre 2015.

38 Paroisse catholique située à $120 \mathrm{~km}$ de la ville de Kikwit et non loin du village natal de Mulele.

39 Curé de la paroisse de Totshi et témoin oculaire de la rébellion muleliste.

40 Archives des Annonciades d'Heverlee, Rapport de la mission catholique de Totshi au Commissaire extraordinaire : 8.2.5.6/1. Correspondances, 1964.

41 Archives des Annonciades d'Heverlee, Correspondances sur la rébellion muleliste : 8.2.5.10/1. Dossier n² $2,1964$.

42 Cette paroisse catholique se situe au sud du Kwilu. Elle fut aussi fortement endommagée par l'insurrection.

43 Témoin n4, R. K. Kikwit, 5 décembre 2015.

44 Témoin n ${ }^{\circ} 6$, M. S. Kikwit, 8 décembre 2015.

45 Antippas, Itinéraires et témoignages. Congo-Zaïre 1960-1980, 186-187.

46 Pour plus de détails, voir Renée Fox et al., "La deuxième indépendance "; Verhaegen, Mulele et la révolution populaire au Kwilu.

47 Marc Depaepe, "Demythologizing the Educational Past : An Endless Task in History of Education ", Historical Studies in Education/Revue d'histoire de l'éducation 9, 2 (1997) : 210.

48 Marc Depaepe and Karen Hulstaert, "Demythologizing the Educational Past : An Attempt to Assess the Power Education in the Congo (DRC) with a Nod to the History of Interwar Pedagogy in Catholic Flanders ", Paedagogica Historica 51, 1-2 (2015) : 19. Traduction libre. [Independence entailed political, and, to a lesser extent, economic independence, but cultural independence, or the so-called decolonization of the mind, would prove much more difficult to achieve].

49 De nombreux écrits ont bien exprimé ce point de vue. À titre indicatif, nous renvoyons à Martin Ekwa, L'école trahie (Kinshasa : Cadicec, 2004); Marc Depaepe, « Rien ne va plus... The Collapse of the Colonial Educational Structures in Zaïre (1960-1965) ", Education and Society. International Journal in Education and Sociology 16,1 (1998) : 37-53 ; Pierre Erny, Essai sur l'éducation en Afrique noire (Paris : L'Harmattan, 2001). On peut également se rendre compte de cette réalité en parcourant les différents programmes des cours.

50 Marc Depaepe et Lies Van Rompaey, In het teken van de bevoogding.

51 Marvin D. Markowitz, "The Missions and Political Development in the Congo ", Africa 40, 8 (1970): 246.

52 Voir par exemple Bajana Kadima-Tshimanga et Léon Michel Ilunga Kongolo, «L'évolué congolais et la Révolution de 1959 au Congo belge. Analyse des textes ", dans Révolte et Société, Tome 1 (Paris : Publications de la Sorbonne, 1989), 177 : «Il s'ensuit que l'unité posée par l'action coloniale au Congo belge demeure une unité formelle. Les contradictions et les incohérences de cette action obligent les intellectuels noirs à adopter une attitude de refus. Refus de l'autre, refus de son mode de vie et surtout refus de se laisser exploiter. Ce triple refus transparaît dans les syntagmes ci-après, définitoires du Nègre : originaire de son pays, être mieux dans son milieu que dans le milieu européen, loin d'atteindre l'équilibre social, essentiellement solidaire, un esprit 
solidaire. » Pour une analyse plus profonde du phénomène de l'évolué (et de l'inégalité sexuelle dans ce contexte), voir également : Jean-Marie Mutamba Makombo Kitatshima, Du Congo belge au Congo indépendant, 1940-1960. Émergence des "évolués " et genèse du nationalisme (Kinshasa : Publications de l'Institut de Formation et d'Études Politiques, 1998).

53 Voir également Barbara A. Yates, «African Reactions to Education : the Congolese Case ", Comparative Education Review 15, 2 (1971): 169. L'auteure cite d'autres facteurs qui entrent en compte dans la situation d'enseignement difficile des filles, comme la crainte de perdre la dot.

54 Jacques Vanderlinden et al., Du Congo au Zaïre, 1960-1980 : Essai de bilan (Bruxelles : CRISP, 1985), 99. Les auteurs concluent que « le bilan de l'enseignement zaïrois entre 1960 et 1980 semble donc très négatif jusqu’à présent : objectifs d'alphabétisation non atteints, mauvais rendements quantitatif et qualitatif, coût financier très élevé ».

55 Kita Masandi, «L'Éducation féminine au Congo belge » : 479.

56 Annuaire statistique du Bureau de l'enseignement catholique, année scolaire 1962-1963.

57 En ce qui concerne le problème des finances, voir : Tom De Herdt et Kristof Titeca, "Governance with Empty Pockets : The Education Sector in the Democratic Republic of Congo ", Development and Change 47 (2006) : 472-494.

58 Barbara A. Yates, "Structural Problems in Education in the Congo (Leopoldville)", Comparative Education Review 7, 2 (1963) : 152-162.

59 Nous faisons ici abstraction de la question de savoir dans quelle mesure cette " tradition congolaise " n'était pas elle-même une invention du colonialisme, voir à ce sujet :

Terence Ranger, "The Invention of Tradition in Colonial Africa », dans Eric Hobsbawn et Terence Ranger (eds.), The Invention of Tradition (Cambridge : Cambridge University Press, 2005), 211-262. Mulele lui-même n'a en aucun cas eu cette réflexion postcoloniale. Nous n'avons pas non plus connaissance de recherches systématiques sur la tradition éducative africaine, bien que certains auteurs aient tenté d'en établir les caractéristiques, par exemple : Michael B. Adeyemi et Augustus A. Adeyinka, "Some Key Issues in African Traditional Education ", McGill Journal of Education 37, 2 (2002) : 223-240.

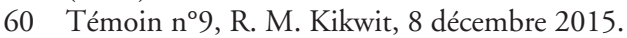

61 Renée Fox et al., " La deuxième indépendance » : 25-26.

62 Ibid., 25-26.

63 Témoin $n^{\circ} 14$, C. N. Kikwit, 9 décembre 2015.

64 Témoin n¹7, G. M. Kikwit, 8 décembre 2015.

65 Témoin n²1, P. L. Kikwit, 12 décembre 2015.

66 Renée Fox et al., "La deuxième indépendance " : 19.

67 Henri Lehalle, Psychologie des adolescents (Paris : PUF, 1987), 205-206.

68 Verhaegen, Mulele et la révolution populaire au Kwilu, 69.

69 Interview $\mathrm{n}^{\circ} 11, \mathrm{~B}$. L. Kikwit, 17 décembre 2015.

70 Ces appellations font allusion à l'éducation par initiation organisée chez les différents peuples du Kwilu en vue de préparer les filles aux responsabilités de femme, d'épouse et de mère.

71 Témoin n², B. S, Kikwit, 1 décembre 2015.

72 Martens, Une femme du Congo, 84.

73 Témoin n 19, F. K. Kikwit, 19 décembre 2015.

74 Martens, Une femme du Congo, 130.

75 Dans un contexte plus large, le mot " malongi » signifie traditionnellement "éducation " dans certaines langues du Kwilu. Mais dans le maquis, il est probable que l'usage de ce mot provient de la réminiscence du catéchisme catholique. 
76 Dans le maquis, les partisans ne pouvaient désigner Pierre Mulele par son nom en guise de respect et de discrétion. C'est ainsi qu'ils utilisaient quelques surnoms symboliques pour faire allusion à leur leader. Dans nos recherches, nous en avons noté cinq avec leur tradition littérale : "Ngo » (léopard en plusieurs langues); en langue kibunda : "Ebun Emvul » (le nuage qui porte la pluie); «Egnang Lamvul » (la goutte de pluie); « Nga Maan " (le chef de terre) et " Nga Bul " (le chef du pays).

77 Interview n ${ }^{\circ}$ 26, M. K. Kikwit, 23 décembre 2015.

78 Il s'agit très probablement du petit livre rouge de Mao édité en 1964 que Mulele aurait pu apporter de la Chine comme symbole de la révolution maoïste.

79 Témoin $\mathrm{n}^{\circ} 12$, E. M. Kikwit, 3 décembre 2015.

80 David Van Reybrouck, Congo. Een geschiedenis (Amsterdam : De Bezige Bij, 2010), 339.

81 Philippe Meirieu, Le choix d'éduquer. Éthique et pédagogie (Paris : ESF, 1991), 145.

82 Sorte de fusil traditionnel.

83 Jacques Chevrier, L'arbre à palabres (Paris : Hatier, 1986), 3.

84 Pierre Erny, Les premiers pas dans la vie de l'enfant d'Afrique Noire. Naissance et première enfance (Paris : L'Harmattan, 1972), 167.

85 Pierre François Edongo, Ethno-anthropologie des punitions en Afrique (Paris : L'Harmattan, 2010), 207.

86 Renée Fox et al., "La deuxième indépendance " : 18.

87 Olenga, "La décolonisation de l'esprit » 14.

88 Précisons tout de même qu'il était strictement interdit aux maquisards de s'exprimer en Lingala. Cependant, le signal populaire des rebelles à l'attaque était «maï-mulele». " Maï » est un mot lingala qui se traduit littéralement par " eau ».

89 Interview n ${ }^{\circ} 7$, E. G. Kikwit, 5 décembre 2015.

90 Jean-Paul Yawidi, Procès de la société congolaise (Kinshasa : Mabiki, 2008), 141.

91 Bajana Kadima-Tshimanga et Léon Michel Ilunga Kongolo, "L'évolué congolais », 176, 178. Les auteurs voient une série de couples antonymiques apportés par la (néo) colonisation : l'être à peau d'ébène vs l'homme blanc; le colonisé vs le colonisateur; des antilopes vs des léopards; des faibles vs des forts, et plus loin les Noirs des grands centres vs les Noirs de l'intérieur et le Noir instruit vs le Noir primitif. Évidemment, on pourrait ici ajouter Homme congolais vs Femme congolaise...

92 Marc Poncelet, Géraldine André et Tom De Herdt, "La survie de l'école primaire congolaise (RDC) : héritage colonial, hybridité et résilience ", Autrepart 2, 54 (2010) : $23-41$.

93 Senghor conçoit le brassage des cultures comme un « rendez-vous du donner et du recevoir » entre les civilisations africaines, occidentales et arabes. Voir Pierre Dumont, Corinne Mencé-Caster et Raphaël Confiant, Senghoriana : éloge à l'un des pères de la négritude (Paris : Publibook, 2011).

94 Patrick Mérand, La vie quotidienne en Afrique noire (Paris : L'Harmattan, 1977), 56.

95 Tom De Herdt and Kristof Titeca, "Governance with Empty Pockets ".

96 Depaepe and Hulstaert, " Demythologising " : 26-29. 